\title{
Article \\ Solution Approach for Bus Transit Model with a Rectangular Service Area
}

Yi-Fong Lin (1)

Citation: Lin, Y.-F. Solution

Approach for Bus Transit Model with

a Rectangular Service Area.

Mathematics 2022, 10, 514. https://

doi.org/10.3390/math10030514

Academic Editor: Ioannis

G. Tsoulos

Received: 17 November 2021

Accepted: 9 December 2021

Published: 5 February 2022

Publisher's Note: MDPI stays neutral with regard to jurisdictional claims in published maps and institutional affiliations.

Copyright: (C) 2022 by the author. Licensee MDPI, Basel, Switzerland. This article is an open access article distributed under the terms and conditions of the Creative Commons Attribution (CC BY) license (https:// creativecommons.org/licenses/by/ $4.0 /)$.
Department of Fashion Industry Management, Hsing Wu University, New Taipei City 25103, Taiwan; 109287@mail.hwu.edu.tw

\begin{abstract}
This paper studies the solution procedure of a bus transit system with a rectangular service area that had been cited more than two hundred times. We will point out that they applied relations suitable for continuous variables, which are not held for a discrete variable and will result in invalid results. We provide our solution procedure to the same example proposed by the original paper to illustrate that their results are less accurate. Our findings will help researchers understand this kind of bus transit system.
\end{abstract}

Keywords: transit bus model; rectangular service zone; optimal solution

\section{Introduction}

Researchers have tried to use a few variables and several constant parameters to describe relationships among variables and parameters, and then build models to explain and predict outcomes of the real world. If there are too many variables in the model, then researchers cannot find the optimal solution, such that in the beginning period of a scientific topic, there are only one or two variables, and then practitioners gradually extend models to include more descriptions and relationships to mimic the real world. The goal of this paper is to study a famous paper by Kocur and Hendrickson [1] that claimed that they obtained a formulated approximated optimal solution for a bus transit system with a rectangular service area. This paper will show that Kocur and Hendrickson derived a less accurate relation between headway and route width, and that their results cannot be applied to a rectangular service area from a city center to a residential housing area that is connected by an (1) express route (no passenger on and off the bus), and (2) bus service route (passenger on and off). Kocur and Hendrickson assumed that the service area has a rectangular shape with length $Y$ and width $X$, where $X$ will be uniformly partitioned into $n$ routes. Hence, the partition number, $n$, with $n \in\{1,2,3, \ldots\}$ is a discrete variable. The other two variables are fare $f$ and headway $h$ (time interval between two adjacent vehicles). Researchers have tried to use calculus to solve problems and then they tend to treat variables as real numbers (a continuous variable), such that they can apply the power tool of derivatives or partial derivatives. Kocur and Hendrickson used route width, $g=\frac{X}{n}$, as a continuous variable. After they derived $f^{*}, h^{*}$, and $g^{*}$, they evaluated $n^{*}=\frac{X}{g^{*}}$ that is not in the desired domain $\{1,2,3, \ldots\}$. For example, in Kocur and Hendrickson, with $X=4, g^{*}=1.31$, they obtained 3.05 bus routes, which is not reasonable. In this paper, we will provide an improvement.

There are four models in Kocur and Hendrickson [1]. We concentrate on the maximum profit model without a vehicle size constraint. They mentioned that the first term of their objective function is the transit revenue, and the second term is the transit operating cost. They provided detailed explanations for the derivations of their objective function. Interested readers, please refer to their original article. Kocur and Hendrickson [1] considered a bus transit model with a rectangular service area. There are three models in Kocur and Hendrickson [1]. Their first model maximized profit without bus capacity constraints. Their second model maximized profit with a bus capacity constraint. Their third model 
maximized profit with user benefit. In this paper, we will focus our attention on their first model.

Since the publication of Kocur and Hendrickson [1], 216 papers have cited this paper in their references. In the following, we provide a brief review of 14 papers among those 216 papers to illustrate the research trend since Kocur and Hendrickson [1]. Furth [2] studied zonal route design for transit corridors to decide zone boundaries and service frequencies, and then solve his problems by dynamic programming. Kuah and Perl [3] developed three bus models to deal with route spacing, headway, and bus-stop spacing. Kuah and Perl [4] considered a feeder-bus network to access a rail rapid transit system under (a) many-to-one, and (b) many-to-many demand patterns, and then they solved their problems by a mathematical programming model. Chang and Schonfeld [5] constructed four bus transit models to minimize cost and maximize profit for a single period and multiple periods with a rectangular service area. Ceder and Israeli [6] applied mixedinteger programming to deal with transit network design problems to allow passengers to have selections of several alternatives. Yang and Bell [7] provided a review paper for the network design problems to discuss model formulations, Braess's paradoxes, and capacity improvement plans. Yan and Chen [8] considered inter-city bus problems with bus route and headway, and then solved them by a mixed-integer multiple flow algorithm. JaraDíaz and Gschwender [9] presented a review paper of microeconomic models with public transport operations concerning the treatment of the variables headway, fleet size, and vehicle size. Gao et al. [10] examined a bilevel transit network model where a traditional transit network design model is the upper model and a transit equilibrium assignment model is the lower model, and then solved this by a heuristic approach. Tirachini and Hensher [11] developed bus transit systems besides headway, vehicle capacity, number of bus routes, and bus stop spacing, they also considered bus fare, and bus operating speed to construct new systems. Lin and Hopscotch [12] examined the first transit model of Imam [13] to show that his model was unreasonable. Owing to Imam [13], who did not provide numerical examples, Lin and Hopscotch [12] adopted numerical data from Kocur and Hendrickson [1] to demonstrate their challenge to Imam [13]. Chen and Julian [14] studied the second transit model of Imam [13] to point out that the solution procedure proposed by Imam [13] contained less accurate results, and then Chen and Julian [14] presented their formulated solutions. Fielbaum et al. [15] used directness, encompassing the number of transfers, number of stops, and passenger route lengths to study scale economies and transit lines in public transport. For interested readers, please refer to Chang and Schonfeld [16] that contains a diagram to describe a bus transit system with a rectangular service area.

Based on the above discussion, papers that cited Kocur and Hendrickson [1] only mentioned the paper in their introduction, and the papers did not pay attention to the solution structure proposed by Kocur and Hendrickson [1], such that they were not aware that the solution approach of Kocur and Hendrickson [1] contained less accurate results. Several related papers are worthy to mention to reveal directions for future studies. Chang and Schonfeld [16] developed a new transit system to include the length of the rectangular service area. Tom and Mohan [17] applied a frequency-coded genetic algorithm to study transit route network design problems. Agrawal and Mathew [18] used a parallel genetic algorithm to examine transit route network design problems. Kepaptsoglou and Karlaftis [19] provided a literature review for transit route network design problems. Mauttone and Urquhart [20] constructed a route setting construction algorithm to solve transit network design problems. Bagloee and Ceder [21] considered transit network design methodology to examine real-world road systems. Ranjbari et al. [22] considered transit network design with elastic demand. Cipriani et al. [23] examined transit network design for a large urban area. Roca-Riu et al. [24] developed urban bus networks among city centers. Xiong et al. [25] constructed a community shuttle model to study routes among metro stations. Yang et al. [26] examined the solution structure of Chang and Schonfeld [16] to point out their less accurate results, and then provided their improvements. Hung and 
Julianne [27] applied the bisection method to find the optimal solution proposed by Yang et al. [26]. Lin and Julian [28] developed a monotonic sequence to replace the alternative sequence proposed by Yang et al. [26], which converges faster than that of Yang et al. [26]. Tung et al. [29] showed that Chang and Schonfeld [16] and Yang et al. [26] focused on the unique solution for real number solutions, such that Chang and Schonfeld [16] and Yang et al. [26] needed an extra condition to guarantee the uniqueness of the critical solution. Tung et al. [29] mentioned that the optimal area length must be a positive number, and then the extra condition proposed by Chang and Schonfeld [16] and Yang et al. [26] becomes redundant. Yang et al. [30] pointed out that if the length of the rectangular service area extends to infinite, the maximum profit will also increase to infinite. Hence, the first transit model proposed by Imam [13] is less accurate. Luo [31] studied the solution procedure of Yang et al. [26], Hung and Julianne [27], and Tung et al. [29] to remove their extra conditions and then provided his new convergent sequence. Wang et al. [32] showed that the formulated solution proposed by Chen and Julian [14] is less accurate, and then they presented their revisions. We will show that the elegant results of an approximated optimal solution proposed by Kocur and Hendrickson [1] are less accurate.

Our paper is organized as follows. We will provide a list of notations in Section 2. We review the solution approach in Section 3. Our improvement is illustrated in Section 4. The same numerical example studied by Kocur and Hendrickson [1] is examined in Section 5. In Section 6, we present a detailed sensitivity analysis for parameters to examine the influence of the fluctuations of parameters. This study points out several directions for future research in Section 7. This paper concludes in Section 8.

\section{Notation}

To be compatible with Kocur and Hendrickson [1], this study uses the same notation as theirs.

Notation is listed as the following:

$a_{1}=$ mode choice coefficient: transit constant

$a_{2}=$ mode choice coefficient: wait and walk time

$a_{3}=$ mode choice coefficient: in-vehicle travel time

$a_{4}=$ mode choice coefficient: fare

$a_{5}=$ mode choice coefficient: auto time and cost

$b=$ spacing between bus stops along a route (mile)

$c=$ bus operating cost (cents/minute)

$d=$ average passenger trip length (mile)

$f=$ bus fare for local service (cents)

$g=X / n$ spacing between parallel bus routes (mile)

$h=$ headway on a local route (minute)

$j=$ average walking speed (mile/minute)

$k=$ the ratio of expected user wait time to headway

$n=$ the partition number for the width

$p=$ trip density by all modes (trips $/ \mathrm{mile}^{2} /$ minute)

$q=$ load factor or ratio of bus passengers to capacity

$s=$ vehicle capacity (passengers/bus)

$T=$ the period of analysis (minute)

$v=$ average local bus speed, including stops (mile/minute)

$X=$ width of the service area (mile)

$Y=$ length of the service area (mile)

\section{Review of Kocur and Hendrickson}

Kocur and Hendrickson [1] converted the objective function from $Q(f, n, h)$ to $Q(f, g, h)$, in which the original variable is " $n$ ", the partition number of the width is a natural number (a discrete variable). Kocur and Hendrickson [1] adopted the route spacing, $g=\frac{X}{n}$ as the 
new variable. They treated $g$ as a continuous variable, and then they considered the partial derivative with respect to $g$.

They tried to maximize $Q(f, g, h)$ as follows,

$$
\mathrm{Q}(f, g, h)=\left(a_{1}+a_{2} \frac{b}{4 j}+\left(\frac{a_{3}}{v}+a_{5}\right) d\right) p T X Y f+a_{2} k p T X Y f h+a_{2} \frac{p}{4 j} T X Y f g+a_{4} p T X Y f^{2}-\frac{2 c T X Y}{v g h} .
$$

Kocur and Hendrickson [1] derived that

$$
\begin{gathered}
\frac{\partial}{\partial f} \mathrm{Q}(f, g, h)=\left(a_{1}+a_{2} \frac{b}{4 j}+\left(\frac{a_{3}}{v}+a_{5}\right) d\right) p T X Y+a_{2} k p T X Y h+a_{2} \frac{p}{4 j} T X Y g+2 a_{4} p T X Y f, \\
\frac{\partial}{\partial g} Q(f, g, h)=a_{2} \frac{p}{4 j} T X Y f+\frac{2 c T X Y}{v g^{2} h},
\end{gathered}
$$

and

$$
\frac{\partial}{\partial h} Q(f, g, h)=a_{2} k p T X Y f+\frac{2 c T X Y}{v g h^{2}} .
$$

Kocur and Hendrickson [1] solved the system of $\frac{\partial}{\partial g} \mathbf{Q}(f, g, h)=0$, and $\frac{\partial}{\partial h} \mathrm{Q}(f, g, h)=0$ to imply

$$
a_{2} \frac{p}{4 j} T X Y f+\frac{2 c T X Y}{v g^{2} h}=0
$$

and

$$
a_{2} k p T X Y f+\frac{2 c T X Y}{v g h^{2}}=0 .
$$

Based on the relations of Equations (5) and (6), they obtained

$$
h^{*}=\frac{1}{4 j k} g^{*} .
$$

They plugged Equation (7) into $\frac{\partial}{\partial g} Q(f, g, h)=0$ to derive

$$
a_{2} \frac{p}{4 j} f+\frac{8 c j k}{v g^{3}}=0
$$

Similarly, they plugged Equation (7) into $\frac{\partial}{\partial f} \mathrm{Q}(f, g, h)=0$ to find

$$
\left(a_{1}+a_{2} \frac{b}{4 j}+\left(\frac{a_{3}}{v}+a_{5}\right) d\right)+a_{2} \frac{g}{2 j}+2 a_{4} f=0 .
$$

Based on Equations (8) and (9) to cancel out $f$, they yielded

$$
\frac{-a_{2}}{2 j A} g^{4}-g^{3}+\frac{64 a_{4} c j^{2} k}{a_{2} p v A}=0 .
$$

with an abbreviation $A$, with $A=a_{1}+a_{2} \frac{b}{4 j}+\left(\frac{a_{3}}{v}+a_{5}\right) d$.

Kocur and Hendrickson [1] mentioned that the coefficient of the first term, $g^{4}$, is relatively small, such that they overlooked the first term of Equation (10) to derive an approximated optimal solution for $g^{*}$ as

$$
g^{*} \approx\left(\frac{64 a_{4} c j^{2} k}{a_{2} p v A}\right)^{1 / 3}
$$

Applying the relation of Equation (7), they obtained an approximated optimal solution for $h^{*}$ as

$$
h^{*} \approx\left(\frac{a_{4} c}{a_{2} j k^{2} p v A}\right)^{1 / 3} .
$$


Referring to the relation of Equation (9), they derived an approximated optimal solution for $f^{*}$ as

$$
f^{*} \approx-\frac{A}{2 a_{4}}-\left(\frac{a_{2}^{2} c k}{a_{4}^{2} j p v A}\right)^{1 / 3}
$$

Based on Equations (11)-(13), Kocur and Hendrickson [1] obtained their approximation solutions for the bus fare, route spacing, and headway.

\section{Our Improvement}

This paper will reconsider the original objective function, $\mathrm{Q}\left(f, \frac{X}{n}, h\right)$, where fare " $f$ " and headway " $h$ " are treated as continuous variables and the partition number, " $n$ ", is treated as a discrete variable. This study recalls the original objective function as follows,

$\mathrm{Q}\left(f, \frac{X}{n}, h\right)=\left(a_{1}+a_{2} \frac{b}{4 j}+\left(\frac{a_{3}}{v}+a_{5}\right) d\right) p T X Y f+a_{2} k p T X Y f h+a_{2} \frac{p}{4 j n} T X^{2} Y f+a_{4} p T X Y f^{2}-\frac{2 c n T Y}{v h}$.

In the following derivation, we will treat " $n$ " as a given natural number that is considered as a constant for the moment.

For a given $n$, we compute $\frac{\partial}{\partial f} \mathrm{Q}\left(f, \frac{X}{n}, h\right)$ and $\frac{\partial}{\partial h} \mathrm{Q}\left(f, \frac{X}{n}, h\right)$ to derive

$$
\begin{aligned}
\frac{\partial}{\partial f} \mathrm{Q}\left(f, \frac{X}{n}, h\right)= & \left(a_{1}+a_{2} \frac{b}{4 j}+\left(\frac{a_{3}}{v}+a_{5}\right) d\right) p T X Y+a_{2} k p T X Y h+a_{2} \frac{p}{4 j n} T X^{2} Y+2 a_{4} p T X Y f, \\
& \text { and } \\
& \frac{\partial}{\partial h} \mathrm{Q}\left(f, \frac{X}{n}, h\right)=a_{2} k p T X Y f+\frac{2 c n T Y}{v h^{2}} .
\end{aligned}
$$

We solve the system of $\frac{\partial}{\partial f} \mathrm{Q}\left(f, \frac{X}{n}, h\right)=0$ and $\frac{\partial}{\partial h} \mathrm{Q}\left(f, \frac{X}{n}, h\right)=0$ to obtain the optimal fare and headway concerning this given " $n$ ". To clearly express our derivation, we will use " $f(n)$ " and " $h(n)$ " to denote our findings concerning this " $n$ ". We obtain

$$
\left(a_{1}+\frac{a_{2}}{4 j}\left(b+\frac{X}{n}\right)+\left(\frac{a_{3}}{v}+a_{5}\right) d\right)+a_{2} k h(n)+2 a_{4} f(n)=0,
$$

and

$$
a_{2} k p f(n)+\frac{2 c n}{v(h(n))^{2} X}=0 .
$$

From Equations (17) and (18), we cancel out $\mathrm{f}$ to derive a relation only containing $h(n)$ as follows,

$$
a_{2}^{2} k^{2} p v X(h(n))^{3}+a_{2} k p v B_{n} X(h(n))^{2}-4 a_{4} c n=0 .
$$

with an abbreviation $B_{n}$, with $B_{n}=a_{1}+\frac{a_{2}}{4 j}\left(b+\frac{X}{n}\right)+\left(\frac{a_{3}}{v}+a_{5}\right) d$.

We assume a series of auxiliary functions as $\mathrm{G}_{n}(y)$, for $n=1,2, \ldots$,

$$
\mathrm{G}_{n}(y)=a_{2}^{2} k^{2} p v X y^{3}+a_{2} k p v B_{n} X y^{2}-4 a_{4} c n,
$$

for any real number $y$. We extend the domain of auxiliary functions from positive numbers (headway) to the real number, such that the following discussion becomes easy to execute.

We derive

$$
\frac{\mathrm{dG}_{n}(y)}{\mathrm{d} y}=3 a_{2}^{2} k^{2} p v X y^{2}+2 a_{2} k p v B_{n} X y,
$$

and

$$
\frac{\mathrm{d}^{2} \mathrm{G}_{n}(y)}{\mathrm{d} y^{2}}=6 a_{2}^{2} k^{2} p v X y+2 a_{2} k p v B_{n} X
$$


Based on Equation (21), we solve $\frac{\mathrm{dG}_{n}(y)}{\mathrm{d} y}=0$ to find two solutions for candidates for local critical points: $y=0$ and $y=\frac{2 B_{n}}{-3 a_{2} k}$. Moreover, based on Equation (22), we solve $\frac{\mathrm{d}^{2} \mathrm{G}_{n}(y)}{\mathrm{d} y^{2}}=0$ to find candidates for inflection point: $y=\frac{B_{n}}{-3 a_{2} k}$.

From our discussion for the inflection point, we know that $G_{n}(y)$ is concave down for $-\infty<y<\frac{B_{n}}{-3 a_{2} k}$ and concave up for $\frac{B_{n}}{-3 a_{2} k}<y<\infty$, since $a_{2}=-0.0081$.

Recalling our discussion for the critical points, we derive that $G_{n}(y)$ increases for $-\infty<y<0$; decreases for $0<y<\frac{2 B_{n}}{-3 a_{2} k}$; increases for $\frac{2 B_{n}}{-3 a_{2} k}<y<\infty$. We sketch the figure for $\mathrm{G}_{n}(y)$ with Cases I and II in the following Figure 1.

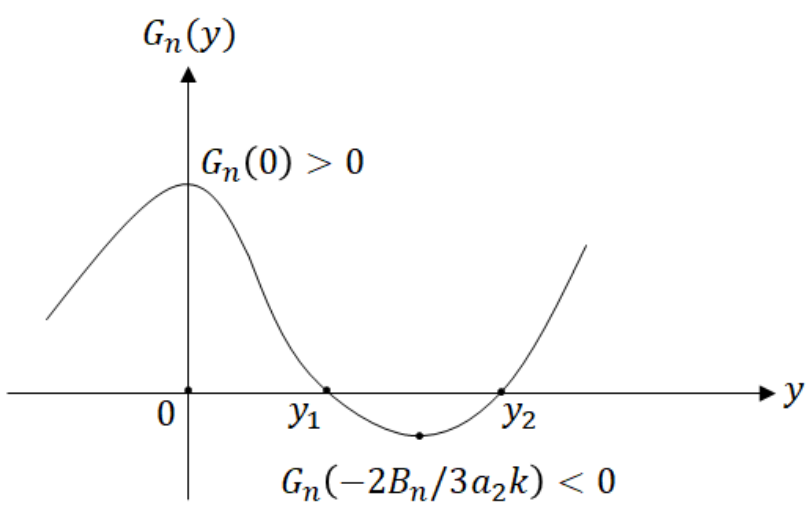

Case I

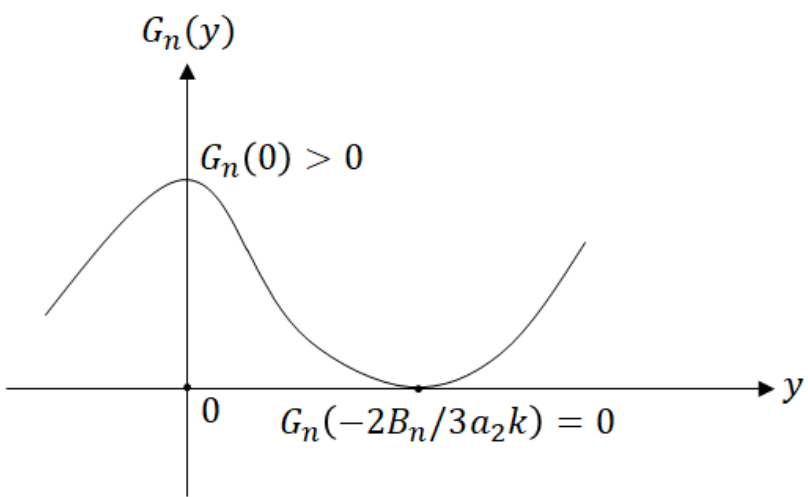

\section{Case II}

Figure 1. Graph of $\mathrm{G}_{n}(y)$ for Cases I and II.

Based on Equation (20), and $a_{4}=-0.0014$, we know that

$$
G_{n}(0)=-4 a_{4} c n>0,
$$

and

$$
G_{n}\left(\frac{2 B_{n}}{-3 a_{2} k}\right)=\frac{1}{a_{2} k} p v X\left(\frac{4 B_{n}^{3}}{27}\right)-4 a_{4} c n,
$$

We need an extra condition

$$
\frac{1}{a_{2} k} p v X\left(\frac{4 B_{n}^{3}}{27}\right)-4 a_{4} c n \leq 0
$$

to ensure the existence of the critical points. In the next section, we will show that our extra condition of Equation (25) is supported by the numerical data in Kocur and Hendrickson [1] for $n=1,2, \ldots, 8$. 
Hence, under our extra condition of Equation (25), we divide the discussions into two cases: Case I: $\frac{1}{a_{2} k} p v X\left(\frac{4 B_{n}^{3}}{27}\right)-4 a_{4} c n<0$, and Case II: $\frac{1}{a_{2} k} p v X\left(\frac{4 B_{n}^{3}}{27}\right)-4 a_{4} c n=0$.

For Case I, there are two positive solutions, denoted as $y_{1}$ and $y_{2}$ that satisfy $\mathrm{G}\left(y_{1}\right)=0$ and $\mathrm{G}\left(y_{2}\right)=0$, with $0<y_{1}<\frac{2 B_{n}}{-3 a_{2} k}<y_{2}$.

For Case II, there is only one point, $\frac{2 B_{n}}{-3 a_{2} k}$ that satisfies $\mathrm{G}(y)=0$.

For a given $n$, under the condition $\frac{1}{a_{2} k} p v X\left(\frac{4 B_{n}^{3}}{27}\right)-4 a_{4} c n<0$, we know that $h(n)$ has two positive solutions that will be denoted as $h_{1}(n)$ and $h_{2}(n)$, such that

$$
0<h_{1}(n)<\frac{2 B_{n}}{-3 a_{2} k}<h_{2}(n)
$$

Refer to Equation (18), we find $f_{1}(n)$ and $f_{2}(n)$, with

$$
f_{1}(n)=\frac{-2 c n}{a_{2} k p v\left(h_{1}(n)\right)^{2} X},
$$

and

$$
f_{2}(n)=\frac{-2 c n}{a_{2} k p v\left(h_{2}(n)\right)^{2} X} .
$$

We will compare $\mathrm{Q}\left(f_{1}(n), X / n, h_{1}(n)\right)$ and $\mathrm{Q}\left(f_{2}(n), X / n, h_{2}(n)\right)$ to find the local maximum value for the given $n$, as follows

$$
\max \left\{\mathrm{Q}\left(f_{1}(n), X / n, h_{1}(n)\right), \mathrm{Q}\left(f_{2}(n), X / n, h_{2}(n)\right)\right\} .
$$

To find the global maximum value, we compute the above results for $n=1,2, \ldots$,

$$
\max _{n=1,2, \ldots} \max \left\{\mathrm{Q}\left(f_{1}(n), X / n, h_{1}(n)\right), \mathrm{Q}\left(f_{2}(n), X / n, h_{2}(n)\right)\right\}
$$

\section{Numerical Example}

We consider the same numerical example proposed by Kocur and Hendrickson [1] with the following data: $a_{1}=0.38, a_{2}=-0.0081, a_{3}=-0.0033, a_{4}=-0.0014, a_{5}=0.0328$, $b=0.2, c=0.5, d=3, j=0.05, k=0.4, p=3.59, T=60, v=0.167, X=4$, and $Y=6$.

To check our extra condition of Equation (25), we assume that $\Delta_{n}=\frac{1}{a_{2} k} p v X\left(\frac{4 B_{n}^{3}}{27}\right)$, $\Phi_{n}=4 a_{4} c n$, and $\Omega_{n}=\frac{1}{a_{2} k} p v X\left(\frac{4 B_{n}^{3}}{27}\right)-4 a_{4} c n$. We list the results in Table 1.

Table 1. Numerical examination for our extra condition.

\begin{tabular}{ccccc}
\hline $\boldsymbol{n}$ & $\boldsymbol{B}_{\boldsymbol{n}}$ & $\boldsymbol{\Delta}_{\boldsymbol{n}}$ & $\boldsymbol{\Phi}_{\boldsymbol{n}}$ & $\mathbf{\Omega}_{\boldsymbol{n}}$ \\
\hline 1 & 0.249 & -1.193 & -0.28 & -1.413 \\
\hline 2 & 0.330 & -3.941 & -0.56 & -3.381 \\
\hline 3 & 0.357 & -4.990 & -0.84 & -4.150 \\
\hline 4 & 0.371 & -5.578 & -1.12 & -4.458 \\
\hline 5 & 0.379 & -5.952 & -1.40 & -4.552 \\
\hline 6 & 0.384 & -6.210 & -1.68 & -4.530 \\
\hline 7 & 0.388 & -6.399 & -1.96 & -4.439 \\
\hline 8 & 0.391 & -6.543 & -2.24 & -4.303 \\
\hline
\end{tabular}

The width of the rectangular service area is 4 miles. The comfortable walking distance to a bus line is 0.25 miles. We compute that $4 / 2(0.25)=8$. Therefore, we stop our examination when $n=8$. 
Based on the findings of Table 1, we know that our extra condition of Equation (25) is supported by the data proposed by Kocur and Hendrickson [1].

For $n=1,2, \ldots, 6$, we find $h_{1}(n)$ and $h_{2}(n)$ that satisfy $\mathrm{G}\left(h_{1}(n)\right)=0$ and $\mathrm{G}\left(h_{2}(n)\right)=0$, with $0<h_{1}(n)<\frac{2 B_{n}}{-3 a_{2} k}<h_{2}(n)$.

For this given $n$, we plug $h_{1}(n)$ into Equation (18), to obtain $f_{1}(n)$ and then refer to Equation (14) to evaluate $\mathrm{Q}\left(f_{1}(n), \mathrm{X} / n, h_{1}(n)\right)$.

Similarly, for $h_{2}(n)$, using Equation (18), we derive $f_{2}(n)$ and then refer to Equation (14) to find $\mathrm{Q}\left(f_{2}(n), X / n, h_{2}(n)\right)$.

We compare $\mathrm{Q}\left(f_{1}(n), X / n, h_{1}(n)\right)$ and $\mathrm{Q}\left(f_{2}(n), X / n, h_{2}(n)\right)$ to select the maximum value which is the local optimal solution for the given $n$.

For $n=1,2, \ldots, 6$, we list our results in the next table.

From Table 2, we can predict that when $n=7,8, \ldots$, the values of $\mathrm{Q}\left(f_{1}(n), \frac{X}{n}, h_{1}(n)\right)$ will gradually decrease and then we can accept that the first local maximum value is the global maximum value. Hence, we stop our computation at $n=6$.

Table 2. Our results for $n=1,2, \ldots, 6$.

\begin{tabular}{ccccccc}
\hline$n$ & $\boldsymbol{h}_{1}(n)$ & $f_{1}(n)$ & $\mathrm{Q}\left(f_{1}(n), X / n, h_{1}(n)\right)$ & $h_{2}(n)$ & $f_{2}(n)$ & $\mathrm{Q}\left(f_{2}(n), X / n, h_{2}(n)\right)$ \\
\hline 1 & 13.22 & 73.64 & $22,938.91$ & 74.87 & 2.30 & -2840.96 \\
\hline 2 & 16.11 & 99.18 & $44,490.79$ & 99.62 & 2.59 & -4279.30 \\
\hline 3 & 19.14 & 105.40 & $46,550.98$ & 107.29 & 3.35 & -5946.10 \\
\hline 4 & 21.94 & 106.95 & $43,467.56$ & 110.73 & 4.20 & -7659.67 \\
\hline 5 & 24.54 & 106.86 & $38,668.11$ & 112.46 & 5.09 & -9396.84 \\
\hline 6 & 27.00 & 105.93 & $33,272.48$ & 113.33 & 6.01 & $-11,151.35$ \\
\hline
\end{tabular}

We find that the optimal solution for this bus transit system is as follows: three bus routes, $n^{*}=3$; headway, $h^{*}=19.14 \mathrm{~min}$; fare, $f^{*}=105.40$ cents.

For completeness, we compare our results with that of Kocur and Hendrickson [1]. They derived route spacing $g^{*}=1.31$ miles, $h^{*}=16.4 \mathrm{~min}$, and $F=109$ cents. However, we find that if

$$
g^{*}=1.31
$$

to indicate that there are 3.053 routes in this rectangular service area, since

$$
n^{*}=\frac{X}{g^{*}}=3.053 \text {. }
$$

Hence, we know that the optimal solution proposed by Kocur and Hendrickson [1] is not reasonable.

Lastly, we recall the relation between the headway, $h(n)$ and the route width, $X / n$. We recall Equation (7) to show that Kocur and Hendrickson [1] believed that the following relation is valid,

$$
h^{*}=\frac{X}{4 j k n^{*}} .
$$

For a given $n$, we compare $h_{1}(n)$ and $X / 4 j k n$ to list the comparison in Table 3.

Table 3. The comparison between $h_{1}(n)$ and $X / 4 j k n$.

\begin{tabular}{ccccccc}
\hline $\boldsymbol{n}$ & $\mathbf{1}$ & $\mathbf{2}$ & $\mathbf{3}$ & $\mathbf{4}$ & $\mathbf{5}$ & $\mathbf{6}$ \\
\hline$h_{1}(n)$ & 13.22 & 16.11 & 19.14 & 21.94 & 24.54 & 27.00 \\
\hline$X / 4 j k n$ & 50 & 25 & 16.67 & 12.5 & 10 & 8.33 \\
\hline
\end{tabular}


We know that $\left\{h_{1}(n), n=1,2, \ldots, 6\right\}$ is the candidate for $h^{*}$. From Table 3 , we find that $h_{1}(n) \neq X / 4 j k n$, for $n=1,2, \ldots, 6$. Hence, Equation (33) has no chance to be valid. Moreover, in the following, we explain the managerial point of view to claim that Equation (33) is not reasonable. From Table 3, our findings of $h_{1}(n)$ increasing with $n$ is reasonable because if there are more routes inside the rectangular bus service area, then the headway should also increase to avoid too many buses being crowded in this rectangular area. On the contrary, the relation of $X / 4 j k n$ decreasing with respect to $n$ will result in too many buses being crowded in this rectangular area.

\section{Sensitivity Analysis}

To study the influence of variation of parameters, we execute a sensitivity analysis for this bus transit model. We will change the values from decreasing 25\%, 15\%, and 5\%, to increasing $5 \%, 15 \%$, and $25 \%$ for the spacing between bus stops along a route $b$, the bus operating $\operatorname{cost} c$, the average passenger trip length $d$, the average walking speed $j$, the ratio of expected user wait time to headway $k$, the trip density by all modes $p$, the period of analysis $T$, the average local bus speed, including stops $v$, the width of service area $X$, and the length of the service area $Y$. We list the results for the variation of the spacing between bus stops along a route in Table 4.

Table 4. Variation of the spacing between bus stops along a route $b$.

\begin{tabular}{cccccccc}
\hline $\boldsymbol{b}$ & $\mathbf{- 2 5 \%}$ & $\mathbf{- 1 5 \%}$ & $\mathbf{- 5 \%}$ & Base & $\mathbf{+ 5 \%}$ & $\mathbf{+ 1 5 \%}$ & $\mathbf{+ 2 5 \%}$ \\
\hline$n^{*}$ & 3 & 3 & 3 & 3 & 3 & 3 & 19.16 \\
\hline$h_{1}\left(n^{*}\right)$ & 19.07 & 19.10 & 19.13 & 19.14 & 19.16 & 19.19 \\
\hline$f_{1}\left(n^{*}\right)$ & 106.17 & 105.84 & 105.51 & 105.40 & 105.18 & 104.85 & 104.52 \\
\hline $\mathrm{Q}\left(f_{1}\left(n^{*}\right), \frac{X}{n^{*}}, h_{1}\left(n^{*}\right)\right)$ & $47,658.11$ & $47,214.25$ & $46,701.74$ & $46,550.98$ & $46,330.58$ & $45,860.77$ & $45,452.32$ \\
\hline
\end{tabular}

Based on Table 4, we know that $n^{*}$ is independent of the spacing between bus stops along a route. $h_{1}\left(n^{*}\right)$ has a positive relationship with the spacing between bus stops along a route. $f_{1}\left(n^{*}\right)$ and $\mathrm{Q}\left(f_{1}\left(n^{*}\right), \frac{X}{n^{*}}, h_{1}\left(n^{*}\right)\right)$ have a negative relationship with the spacing between bus stops along a route. We list the results for variation of the bus operating cost in Table 5.

Table 5. Variation of the bus operating $\operatorname{cost} c$.

\begin{tabular}{cccccccc}
\hline$c$ & $-\mathbf{2 5 \%}$ & $\mathbf{- 1 5 \%}$ & $\mathbf{- 5 \%}$ & Base & $\mathbf{+ 5 \%}$ & $\mathbf{+ 1 5 \%}$ & $\mathbf{+ 2 5 \%}$ \\
\hline$n^{*}$ & 3 & 3 & 3 & 3 & 3 & 3 & 21.71 \\
\hline$h_{1}\left(n^{*}\right)$ & 16.33 & 17.49 & 18.60 & 19.14 & 19.67 & 20.71 & 103.52 \\
\hline$f_{1}\left(n^{*}\right)$ & 108.59 & 107.29 & 106.02 & 105.40 & 104.78 & 102.71 \\
\hline $\mathrm{Q}\left(f_{1}\left(n^{*}\right), \frac{X}{n^{*}}, h_{1}\left(n^{*}\right)\right)$ & $55,672.79$ & $51,847.94$ & $48,264.25$ & $46,550.98$ & $44,884.92$ & $41,681.71$ & $38,632.39$ \\
\hline
\end{tabular}

Based on Table 5, we know that $n^{*}$ is independent of the bus operating cost. $h_{1}\left(n^{*}\right)$ has a positive relationship with the bus operating cost. $f_{1}\left(n^{*}\right)$ and $\mathrm{Q}\left(f_{1}\left(n^{*}\right), \frac{X}{n^{*}}, h_{1}\left(n^{*}\right)\right)$ have a negative relationship with the bus operating cost. We list the results for variation of the average passenger trip length in Table 6. 
Table 6. Variation of the average passenger trip length $d$.

\begin{tabular}{cccccccc}
\hline$d$ & $-\mathbf{2 5 \%}$ & $\mathbf{- 1 5 \%}$ & $\mathbf{- 5 \%}$ & Base & $\mathbf{+ 5 \%}$ & $\mathbf{+ 1 5 \%}$ & $\mathbf{+ 2 5 \%}$ \\
\hline$n^{*}$ & 3 & 3 & 3 & 3 & 3 & 3 & 18.80 \\
\hline$h_{1}\left(n^{*}\right)$ & 19.51 & 19.36 & 19.22 & 19.14 & 19.07 & 18.93 & 107.75 \\
\hline$f_{1}\left(n^{*}\right)$ & 101.44 & 103.01 & 104.52 & 105.40 & 106.17 & 109.24 \\
\hline $\mathrm{Q}\left(f_{1}\left(n^{*}\right), \frac{X}{n^{*}}, h_{1}\left(n^{*}\right)\right)$ & $41,323.48$ & $43,390.72$ & $45,489.64$ & $46,550.98$ & $47,620.20$ & $49,782.28$ & $51,975.84$ \\
\hline
\end{tabular}

Based on Table 6, we know that $n^{*}$ is independent of the average passenger trip length. $h_{1}\left(n^{*}\right)$ has a negative relationship with the average passenger trip length. $f_{1}\left(n^{*}\right)$ and $\mathrm{Q}\left(f_{1}\left(n^{*}\right), \frac{X}{n^{*}}, h_{1}\left(n^{*}\right)\right)$ have a positive relationship with the average passenger trip length. We list the results for variation of the average walking speed in Table 7.

Table 7. Variation of the average walking speed $j$.

\begin{tabular}{cccccccc}
\hline$j$ & $\mathbf{- 2 5 \%}$ & $\mathbf{- 1 5 \%}$ & $\mathbf{- 5 \%}$ & Base & $\mathbf{+ 5 \%}$ & $\mathbf{+ 1 5 \%}$ & $\mathbf{+ 2 5 \%}$ \\
\hline$n^{*}$ & 3 & 3 & 3 & 3 & 3 & 3 & 18.71 \\
\hline$h_{1}\left(n^{*}\right)$ & 19.95 & 19.56 & 19.26 & 19.14 & 19.04 & 18.86 & 108.55 \\
\hline$f_{1}\left(n^{*}\right)$ & 97.01 & 100.92 & 104.10 & 105.40 & 106.51 & 110.30 \\
\hline $\mathrm{Q}\left(f_{1}\left(n^{*}\right), \frac{X}{n^{*}}, h_{1}\left(n^{*}\right)\right)$ & $35,721.11$ & $40,706.55$ & $44,781.89$ & $46,550.98$ & $48,170.58$ & $51,030.14$ & $53,474.14$ \\
\hline
\end{tabular}

Based on Table 7, we know that $n^{*}$ is independent of the average walking speed. $h_{1}\left(n^{*}\right)$ has a negative relationship with the average walking speed. $f_{1}\left(n^{*}\right)$ and $\mathrm{Q}\left(f_{1}\left(n^{*}\right), \frac{X}{n^{*}}, h_{1}\left(n^{*}\right)\right)$ have a positive relationship with the average walking speed. We list the results for variation of the ratio of expected user wait time to headway in Table 8.

Table 8. Variation of the ratio of expected user wait time to headway $k$.

\begin{tabular}{cccccccc}
\hline$k$ & $-\mathbf{2 5 \%}$ & $\mathbf{- 1 5 \%}$ & $\mathbf{- 5 \%}$ & Base & $\mathbf{+ 5 \%}$ & $\mathbf{+ 1 5 \%}$ & $\mathbf{+ 2 5 \%}$ \\
\hline$n^{*}$ & 3 & 3 & 3 & 3 & 3 & 3 & 17.37 \\
\hline$h_{1}\left(n^{*}\right)$ & 21.77 & 20.58 & 19.58 & 19.14 & 18.74 & 18.01 & 103.51 \\
\hline$f_{1}\left(n^{*}\right)$ & 108.62 & 107.25 & 106.01 & 105.40 & 104.71 & 102.38 \\
\hline $\mathrm{Q}\left(f_{1}\left(n^{*}\right), \frac{X}{n^{*}}, h_{1}\left(n^{*}\right)\right)$ & $55,672.79$ & $51,847.94$ & $48,264.26$ & $46,550.98$ & $44,884.93$ & $41,681.70$ & $38,632.39$ \\
\hline
\end{tabular}

Based on Table 8, we know that $n^{*}$ is independent of the ratio of expected user wait time to a headway. $h_{1}\left(n^{*}\right), f_{1}\left(n^{*}\right)$, and $\mathrm{Q}\left(f_{1}\left(n^{*}\right), \frac{X}{n^{*}}, h_{1}\left(n^{*}\right)\right)$ have a negative relationship with the ratio of expected user wait time to a headway. We list the results for variation of the trip density by all modes in Table 9.

Table 9. Variation of the trip density by all modes $p$.

\begin{tabular}{cccccccc}
\hline $\boldsymbol{p}$ & $\mathbf{- 2 5 \%}$ & $\mathbf{- 1 5 \%}$ & $\mathbf{- 5 \%}$ & Base & $\mathbf{+ 5 \%}$ & $\mathbf{+ 1 5 \%}$ & $\mathbf{+ 2 5 \%}$ \\
\hline$n^{*}$ & 3 & 3 & 3 & 3 & 3 & 3 & 16.92 \\
\hline$h_{1}\left(n^{*}\right)$ & 22.53 & 20.98 & 19.70 & 19.14 & 18.63 & 17.71 & 107.05 \\
\hline$f_{1}\left(n^{*}\right)$ & 101.42 & 103.20 & 104.73 & 105.40 & 105.95 & 107.89 \\
\hline $\mathrm{Q}\left(f_{1}\left(n^{*}\right), \frac{X}{n^{*}}, h_{1}\left(n^{*}\right)\right)$ & $27,146.92$ & $34,731.30$ & $42,558.56$ & $46,550.98$ & $50,590.64$ & $58,798.47$ & $67,159.34$ \\
\hline
\end{tabular}


Based on Table 9, we know that $n^{*}$ is independent of the trip density by all modes. $h_{1}\left(n^{*}\right)$ has a negative relationship with the trip density by all modes. $f_{1}\left(n^{*}\right)$ and $Q\left(f_{1}\left(n^{*}\right), \frac{X}{n^{*}}, h_{1}\left(n^{*}\right)\right)$ have a positive relationship with the trip density by all modes. We list the results for variation of the period of analysis in Table 10.

Table 10. Variation of the period of analysis $T$.

\begin{tabular}{cccccccc}
\hline $\boldsymbol{T}$ & $\mathbf{- 2 5 \%}$ & $\mathbf{- 1 5 \%}$ & $\mathbf{- 5 \%}$ & Base & $\mathbf{+ 5 \%}$ & $\mathbf{+ 1 5 \%}$ & $\mathbf{+ 2 5 \%}$ \\
\hline$n^{*}$ & 3 & 3 & 3 & 3 & 3 & 3 & 19.14 \\
\hline$h_{1}\left(n^{*}\right)$ & 19.14 & 19.14 & 19.14 & 19.14 & 19.14 & 19.14 \\
\hline$f_{1}\left(n^{*}\right)$ & 105.40 & 105.40 & 105.40 & 105.40 & 105.40 & 105.40 & 105.40 \\
\hline $\mathrm{Q}\left(f_{1}\left(n^{*}\right), \frac{X}{n^{*}}, h_{1}\left(n^{*}\right)\right)$ & $34,913.23$ & $39,568.33$ & $44,223.43$ & $46,550.98$ & $48,878.53$ & $53,533.62$ & $58,188.72$ \\
\hline
\end{tabular}

Based on Table 10, we know that $n^{*}, h_{1}\left(n^{*}\right)$, and $f_{1}\left(n^{*}\right)$ are independent of the period of analysis. $\mathrm{Q}\left(f_{1}\left(n^{*}\right), \frac{X}{n^{*}}, h_{1}\left(n^{*}\right)\right)$ has a positive relationship with the period of analysis. We list the results for variation of the average local bus speed, including stops in Table 11.

Table 11. Variation of the average local bus speed, including stops $v$.

\begin{tabular}{cccccccc}
\hline$v$ & $-\mathbf{2 5 \%}$ & $\mathbf{- 1 5 \%}$ & $\mathbf{- 5 \%}$ & Base & $\mathbf{+ 5 \%}$ & $\mathbf{+ 1 5 \%}$ & $\mathbf{+ 2 5 \%}$ \\
\hline$n^{*}$ & 2 & 3 & 3 & 3 & 3 & 3 & 16.56 \\
\hline$h_{1}\left(n^{*}\right)$ & 19.75 & 21.42 & 19.82 & 19.14 & 18.53 & 17.47 & 110.01 \\
\hline$f_{1}\left(n^{*}\right)$ & 87.99 & 99.00 & 103.46 & 105.40 & 107.10 & 112.64 \\
\hline $\mathrm{Q}\left(f_{1}\left(n^{*}\right), \frac{X}{n^{*}}, h_{1}\left(n^{*}\right)\right)$ & $26,880.39$ & $35,392.13$ & $43,119.67$ & $46,550.98$ & $49,735.90$ & $55,467.59$ & $60,485.22$ \\
\hline
\end{tabular}

Based on Table 11, we know that $n^{*}$ is non-decreasing with the average local bus speed, including stops. $h_{1}\left(n^{*}\right)$ does not have a monotonic relationship with the average local bus speed, including stops. $f_{1}\left(n^{*}\right)$ and $Q\left(f_{1}\left(n^{*}\right), \frac{X}{n^{*}}, h_{1}\left(n^{*}\right)\right)$ have a positive relationship with the average local bus speed, including stops. We list the results for variation of the width of the service area $X$ in the next Table 12 .

Table 12. Variation of the width of the service area $X$.

\begin{tabular}{cccccccc}
\hline $\boldsymbol{X}$ & $\mathbf{- 2 5 \%}$ & $\mathbf{- 1 5 \%}$ & $\mathbf{- 5 \%}$ & Base & $\mathbf{+ 5 \%}$ & $\mathbf{+ 1 5 \%}$ & $\mathbf{+ 2 5 \%}$ \\
\hline$n^{*}$ & 2 & 2 & 3 & 3 & 3 & 3 & 17.35 \\
\hline$h_{1}\left(n^{*}\right)$ & 18.16 & 17.21 & 19.60 & 19.14 & 18.73 & 17.98 & 103.86 \\
\hline$f_{1}\left(n^{*}\right)$ & 104.07 & 102.24 & 105.80 & 105.40 & 104.82 & 102.61 \\
\hline $\mathrm{Q}\left(f_{1}\left(n^{*}\right), \frac{X}{n^{*}}, h_{1}\left(n^{*}\right)\right)$ & $35,061.89$ & $39,314.72$ & $43,954.18$ & $46,550.98$ & $49,045.71$ & $53,722.56$ & $57,975.62$ \\
\hline
\end{tabular}

Based on Table 12, we know that $n^{*}$ is non-decreasing with the width of the service area. $h_{1}\left(n^{*}\right)$ and $f_{1}\left(n^{*}\right)$ do not have a monotonic relationship with the width of the service area. $\mathrm{Q}\left(f_{1}\left(n^{*}\right), \frac{X}{n^{*}}, h_{1}\left(n^{*}\right)\right)$ has a positive relationship with the width of the service area. We list the results for variation of the length of the service area in Table 13. 
Table 13. Variation of the length of the service area $Y$.

\begin{tabular}{cccccccc}
\hline $\boldsymbol{Y}$ & $\mathbf{- 2 5 \%}$ & $\mathbf{- 1 5 \%}$ & $\mathbf{- 5 \%}$ & Base & $\mathbf{+ 5 \%}$ & $\mathbf{+ 1 5 \%}$ & $\mathbf{+ 2 5 \%}$ \\
\hline$n^{*}$ & 3 & 3 & 3 & 3 & 3 & 3 & 19.14 \\
\hline$h_{1}\left(n^{*}\right)$ & 19.14 & 19.14 & 19.14 & 19.14 & 19.14 & 19.14 & 14.14 \\
\hline$f_{1}\left(n^{*}\right)$ & 105.40 & 105.40 & 105.40 & 105.40 & 105.40 & 105.40 & 105.40 \\
\hline $\mathrm{Q}\left(f_{1}\left(n^{*}\right), \frac{X}{n^{*}}, h_{1}\left(n^{*}\right)\right)$ & $34,913.23$ & $39,568.33$ & $44,223.43$ & $46,550.98$ & $48,878.53$ & $53,533.62$ & $58,188.72$ \\
\hline
\end{tabular}

Based on Table 13, we know that $n^{*}, h_{1}\left(n^{*}\right)$, and $f_{1}\left(n^{*}\right)$ are independent of the length of the service area. $\mathrm{Q}\left(f_{1}\left(n^{*}\right), \frac{X}{n^{*}}, h_{1}\left(n^{*}\right)\right)$ has a positive relationship with the length of the service area.

\section{Direction for Future Research}

Based on Table 2, for a given $n$, we predict that $\mathrm{Q}\left(f_{1}(n), X / n, h_{1}(n)\right)$ is a local maximum value and $\mathrm{Q}\left(f_{2}(n), X / n, h_{2}(n)\right)$ is a local minimum value. To verify our above prediction by Hessian matrix,

$$
\left[\begin{array}{cc}
\frac{\partial^{2} Q}{\partial f^{2}} & \frac{\partial^{2} Q}{\partial h \partial f} \\
\frac{\partial^{2} Q}{\partial f \partial h} & \frac{\partial^{2} Q}{\partial h^{2}}
\end{array}\right]
$$

will be an interesting research topic in the future. Moreover, researchers may consider the following auxiliary function, denoted as $\mathrm{M}(n)$,

$$
\mathrm{M}(n)=\mathrm{Q}\left(f_{1}(n), X / n, h_{1}(n)\right),
$$

for $n=1,2, \ldots$

Under the numerical data proposed by Kocur and Hendrickson [1], to prove that $\mathrm{M}(3)$ is the maximum value of $\mathrm{M}(n)$ for $n=1,2, \ldots$, by an algebraic method to fulfill the gap, we only computed $\mathrm{Q}\left(f_{1}(n), X / n, h_{1}(n)\right)$ for $n=1,2, \ldots, 6$ to find the first local maximum, and then treated this first local maximum as the global maximum.

From Equation (20), for a given $n, \mathrm{G}_{n}(y)$ is a cubic polynomial, such that $\mathrm{G}_{n}(y)=0$ has formulated solutions. Researchers can express $h_{1}(n)$ in a closed-form expression and then by Equation (18), and denote $f_{1}(n)$ in a closed-form expression. Hence, researchers can simplify $\mathrm{Q}\left(f_{1}(n), X / n, h_{1}(n)\right)$ to $\mathrm{Q}(n)$, under the closed-form solution of $f_{1}(n)$ and $h_{1}(n)$. Lastly, solving the maximum problem of $\mathrm{Q}(n)$ will be another research problem in the future.

\section{Conclusions}

We showed that a well-known relationship developed by Kocur and Hendrickson [1], which was accepted by many following papers related to bus transit system, is invalid. This is the main contribution of this paper.

We examined Kocur and Hendrickson [1] to show that their approximated formulated solution was based on a less accurate relationship between headway and the partition number of the width of the rectangular service area. We presented our solution procedure to find the first local maximum, and then numerical data illustrated our result is the global maximum. Several possible improvements were discussed in the direction of future research.

Funding: This research received no external funding.

Institutional Review Board Statement: Not applicable.

Informed Consent Statement: Not applicable. 
Data Availability Statement: The data used to support the findings of this study are available from the corresponding author upon request.

Acknowledgments: The author expressed his sincere appreciation for the kind help from Jason Chou (ts883088@hotmail.com) and Cheng-Tan Tung (tung@mail.cpu.edu.tw).

Conflicts of Interest: The author declares no conflict of interest.

\section{References}

1. Kocur, G.; Hendrickson, C. Design of local bus service with demand equilibration. Transp. Sci. 1982, 16, 149-170. [CrossRef]

2. Furth, P.G. Zonal route design for transit corridors. Transp. Sci. 1986, 20, 1-62. [CrossRef]

3. Kuah, G.K.; Perl, J. Optimization of feeder bus routes and bus-stop spacing. J. Transp. Eng. 1988, 114, 341-354. [CrossRef]

4. Kuah, G.K.; Perl, J. The feeder-bus network-design problem. J. Oper. Res. Soc. 1989, 40, 751-767. [CrossRef]

5. Chang, S.K.; Schonfeld, P.M. Multiple period optimization of bus transit systems. Transp. Res. Part B-Methodol. 1991, 25, 453-478. [CrossRef]

6. Ceder, A.; Israeli, Y. User and operator perspectives in transit network design. Transp. Res. Rec. J. Transp. Res. Board 1998, 1623, 3-7. [CrossRef]

7. Yang, H.; Bell, M.G.H. Models and algorithms for road network design: A review and some new developments. Transp. Rev. 1998, 18, 257-278. [CrossRef]

8. Yan, S.; Chen, H.-L. A scheduling model and a solution algorithm for inter-city bus carriers. Transp. Res. Part A Policy Pract. 2002, 36, 805-825. [CrossRef]

9. Jara-Díaz, S.; Gschwender, A. Towards a general microeconomic model for the operation of public transport. Transp. Rev. 2003, 23, 453-469. [CrossRef]

10. Gao, Z.; Sun, H.; Shan, L.L. A continuous equilibrium network design model and algorithm for transit systems. Transp. Res. Part B-Methodol. 2004, 38, 235-250. [CrossRef]

11. Tirachini, A.; Hensher, D.A. Bus congestion, optimal infrastructure investment and the choice of a fare collection system in dedicated bus corridors. Transp. Res. Part B-Methodol. 2011, 45, 828-844. [CrossRef]

12. Lin, S.-C.; Hopscotch, C. Bus service model with rectangular service zone under exponential relation. Int. J. Inf. Manag. Sci. 2016, $27,61-72$.

13. Imam, M.O. Optimal design of public bus service with demand equilibrium. J. Transp. Eng. 1998, 124, 431-436. [CrossRef]

14. Chen, P.S.; Julian, P. Bus model with a rectangular service zone revisit. J. Transp. Eng. Part A Syst. 2018, 144, 04017080. [CrossRef]

15. Fielbaum, A.; Jara-Diaz, S.; Gschwender, A. Beyond the Mohring effect: Scale economies induced by transit lines structures design. Econ. Transp. 2020, 22, 100163. [CrossRef]

16. Chang, S.-K.; Schonfeld, P.M. Optimal dimensions of bus service zones. J. Transp. Eng. 1993, 119, 567-584. [CrossRef]

17. Tom, V.M.; Mohan, S. Transit route network design using frequency coded genetic algorithm. J. Transp. Eng. 2003, 129, 186-195. [CrossRef]

18. Agrawal, J.; Mathew, T.V. Transit route network design using parallel genetic algorithm. J. Comput. Civ. Eng. 2004, 18, 248-256. [CrossRef]

19. Kepaptsoglou, K.; Karlaftis, M. Transit route network design problem: Review. J. Transp. Eng. 2009, 135, 491-505. [CrossRef]

20. Mauttone, A.; Urquhart, M.E. A route set construction algorithm for the transit network design problem. Comput. Oper. Res. 2009, 36, 2440-2449. [CrossRef]

21. Bagloee, S.A.; Ceder, A. Transit-network design methodology for actual-size road networks. Transp. Res. Part B-Methodol. 2011, 45, 1787-1804. [CrossRef]

22. Ranjbari, A.; Shariat-Mohaymany, A.; Amiripour, S.M.M. Transit network design: The necessity of elastic demand consideration. Appl. Mech. Mater. 2011, 97-98, 1117-1122. [CrossRef]

23. Cipriani, E.; Gori, S.; Petrelli, M. Transit network design: A procedure and an application to a large urban area. Transp. Res. Part C-Emerg. Technol. 2012, 20, 3-14. [CrossRef]

24. Roca-Riu, M.; Estrada, M.; Trapote, C. The design of interurban bus networks in city centers. Transp. Res. Part A Policy Pract. 2012, 46, 1153-1165. [CrossRef]

25. Xiong, J.; Guan, W.; Song, L.; Huang, A.; Shao, C. Optimal routing design of a community shuttle for metro stations. J. Transp. Eng. 2013, 139, 1211-1223. [CrossRef]

26. Yang, G.-K.; Lin, J.; Julian, P. Solution structure for an analytic model of bus service zones. J. Transp. Eng. 2014, $140,06013001$. [CrossRef]

27. Hung, K.-C.; Julianne, P. An Improved Solution Method for Bus Service Zones. J. Interdiscipl. Math. 2015, 18, 629-637. [CrossRef]

28. Lin, S.-C.; Julian, P. Monotonic Approach for Optimal Route Length of Bus Service Zoom Model. J. Interdiscipl. Math. 2015, 18, 417-425. [CrossRef]

29. Tung, C.-T.; Chu, C.-H.; Hung, K.-C.; Deng, P.-S. Improved Analytic Model of the Optimum Dimensions Designated for Transit Bus Service Zones. J. Transp. Eng. 2015, 141, 04014095. [CrossRef]

30. Yang, G.-K.; Tung, C.-T.; Hopscotch, C. Rectangular Bus Service Zone without Capacity Constraint. J. Interdiscipl. Math. 2015, 18, 649-656. [CrossRef] 
31. Luo, X.-R. Transit network models with rectangular service zone revisit. J. Discret. Math. Sci. Cryptogr. 2017, 20, 503-514. [CrossRef]

32. Wang, B.; Lin, E.; Chen, Z. Improved Solution for Traffic Model with a Rectangular Service Zone. Int. J. Sci. Eng. Res. 2019, 10, 72-84. 